\title{
Impacts of Practice Combinations on Organizational Knowledge: Based on March's Exploration-Exploitation Model
}

\author{
Yunhao Gong $\mathbb{D}$, Yun Le $\mathbb{D}$, Xinyue Zhang $\mathbb{D}$, and Xiaoyan Chen $(\mathbb{D}$ \\ School of Economics and Management, Tongji University, Shanghai 200092, China \\ Correspondence should be addressed to Xinyue Zhang; xinyue_cinyea@163.com
}

Received 18 August 2021; Accepted 5 October 2021; Published 18 October 2021

Academic Editor: Hiroki Sayama

Copyright ( 92021 Yunhao Gong et al. This is an open access article distributed under the Creative Commons Attribution License, which permits unrestricted use, distribution, and reproduction in any medium, provided the original work is properly cited.

\begin{abstract}
Organizational learning is an important approach for organizations to improve knowledge levels and enhance adaptability to a complex environment. In this paper, based on the exact recreation of March's classical model on organizational learning, we conduct research systematically on the impacts of different rate combinations of two typical organizational practices, socialization and codification, on the level of organizational knowledge. Environmental dynamism and system openness are taken into account, as contextual variables. The result shows that (1) accelerating codification and slowing down socialization can achieve better outcomes in a stable environment and closed system. (2) Moderate system openness is beneficial for organizational knowledge when in a stable environment. (3) Environmental turbulence has obvious negative effects on organizational knowledge, and the adjustment of rates of socialization and codification only works temporarily, when in the closed system. (4) System openness can relieve the negative correlation between environmental turbulence and organizational knowledge. Furthermore, we discuss some challenges in how to apply research findings in this paper to organizational actual operations and also provide a few suggestions for further studies. Our paper enriches relative literature on March's agent-based model, and some results and conclusions obtained in the paper can provide a helpful reference for follow-up researches in this domain.
\end{abstract}

\section{Introduction}

Organizational adaptability to the open system and changing environment has long been a major topic in both organizational theory and strategic management [1]. The organization makes constant adjustments between the exploitation of old certainties and the exploration of new possibilities through continuous learning to find the "best practices" to enhance adaptation to the turbulent environment [2]. Organizational knowledge, the outcome of organizational learning, is regarded as a vital intermediate variable linking organizational practices and organizational performance [3], though the knowledge itself does not face the market test directly. The knowledge accumulated by the organization guides its actions and commitments according to organizational strategy. Various combinations of rich organizational practices influence the knowledge acquisition of organizations in turn.
Linking managerial actions to organizational performance has been an attractive issue in organizational research. March makes a pathbreaking contribution to solving this problem from the perspective of organizational learning. His pioneering work, "Exploration and Exploitation in Organizational Learning" [2], has well documented that maintaining an appropriate balance between exploratory and exploitive practices is a critical factor for organizational survival and prosperity. The core of March's work is an agent-based model, which reflects the joint learning between the organization and its members: the organization learns from its members, and individuals learn from the organization. Through the method of computational simulation, March realizes the quantitative analysis between organizational practice and knowledge.

March's iconic research has a broad and profound impact in the field of organizational learning, knowledge management, organizational strategy, and innovation [4]. Web of Science reports more than 9200 citations of this 
article, and the number in Google Scholar is even over 27000 (accessed August 6, 2021). In massive citations, studies on March's classic model have formed an important research genre. The genre can be mainly divided into four directions. The first direction is the deeper excavation based on the original model from different perspectives. For example, Chanda [3] reveals, using March's initial model, how to evaluate managers' contributions towards organizational success although circumstances beyond managers' control may affect outcomes, from the complexity perspective. Other studies such as Chanda and Ray [5], Chanda et al. [6], and Chanda and McKelvey [7] all belong to this direction. The second direction is extending the model by adding some real-world features, such as self-experimentation [8], forgetting by individual [9], interpersonal learning [10,11], the amplitude and frequency of environmental turbulence $[1,12]$, and organizational network structure $[12,13]$. The third direction is the specific application of the model, such as in the information technology (IT) industry $[14,15]$ and in parallel problem solving [16]. The fourth one is developing new models, like the NK model $[17,18]$, the multiarm bandit model [19], and so on, under the guidance of March's basic ideas.

Our study in this paper belongs to the first direction above. Despite the growing literature on this direction, there is still a fundamental challenge that deserves our attention. The focus of a majority of existing researches in this direction is seeking to build a new theory, which is called verbal theory $[20,21]$, through various exploration from different dimensions of the original model, but not enough insight is put into the initial model itself. March describes two typical kinds of organizational practice, socialization and codification, in his model. System openness and environmental dynamism are set as contextual variables, and the level of organizational knowledge is set as an outcome variable. Then, what are the impacts of different rate combinations of socialization and codification on the level of organizational knowledge in various contexts? This is a basic question about the model, but it has hardly been studied specifically and systematically in the present literature on this direction, although some aspects of this issue are mentioned in some articles. We deem that the in-depth examination of this fundamental question can give us a fuller understanding of March's classical model and is of great benefit for the development of new theory using the model. In addition, considering that March's original model is the starting point of the research genre $[22,23]$, the solution to this question will provide a new step, to a certain extent, for interrelated investigations in the future, whether their approaches or aims are in-depth explorations, further extension, specific application, or remodeling.

The main content of this paper is organized as follows. First, we illustrate the theoretical basis of this paper. Next, based on the detailed description of March's computational simulation mode, we recreate it exactly. Then, we conduct a comprehensive and systematical examination of the results obtained by the reconstructed model. As stated above, we pay attention to investigating how different practice combinations influence organizational knowledge in different contexts. At last, we list the conclusions of this paper and discuss them from a practical perspective. In addition, some directions for further research based on our study are given.

\section{Theoretical Foundations}

2.1. Organizational Knowledge. Knowledge in organizations consists of two parts, organizational knowledge, and members' knowledge. In terms of the scope attribute of knowledge, the former is collective knowledge, and the latter is individual knowledge. At the level of individuals, knowledge constitutes an understanding of principles, facts, and processes that range from generic to specific [24]. This knowledge is dispersed in an organization in the brains of its employees. Organizational knowledge is the validated understanding and beliefs in an organization about the relationship between the organization and its environment [25]. Standard operating procedures, information technology systems, the physical layout of production and distribution facilities, and so on are all manifestations of organizational knowledge. In March's terminology, the organizational code is the repository for collective knowledge in organizations, so the organizational knowledge is also called code knowledge or organizational code knowledge.

The creation of organizational knowledge is dependent on the ability of organization members to exchange and combine existing information, knowledge, and ideas [26]. At the beginning of the establishment of organizations, they only have individual knowledge, no collective knowledge. During the course of organizational functioning, the members of an organization learn either from the organizational code (that stores procedures, norms, rules, and forms) or from other members [10]. In our model, following March [2], we implement only the first mechanism, that is, we do not incorporate a mechanism for organizational members learning from each other; rather, members learn only from the organizational code. Moreover, the knowledgeable members of the organization also participate in a process we cite as organizational codification (March calls this learning by the organizational code). In organizational codification, members having superior knowledge contribute to updating the knowledge stored in the organizational code. When recruits enter the organization, they are socialized into organizational languages, beliefs, and practices, realizing the growth of individual knowledge and enhancing the adaptation to the organization. On the other hand, new members provide an additional source for organizational learning by facilitating the inflow of knowledge diversity.

This form of mutual learning has effects on both organizational members and the whole organization [27]. Organizational knowledge is shaped by knowledge of the superior members and plays a role in shaping the knowledge of organizational members. Individuals host knowledge regarding the organizational purposes and tasks they are associated with, by learning the best practice from the organization in a specific aspect. During the process, individuals' imperfect imitation of organizational knowledge and self-experimentation in practice will lead to exploitative and exploratory innovations [28], which in turn generate 
new best practices, because organizational best practices are not stationary as the environment changes. For example, in a construction engineering company, through comprehensive and strict induction training, safety officers have a good understanding of the company's mechanical operation standards, safety management system, emergency handling procedures, and so on. However, with the promulgation of new industry safety regulations, the original institution of the company is no longer applicable. Combined with the specific work practice, the safety officer formulates a new safety management system under the guidance of the regulations, which becomes the company's new construction standard after careful consideration and refinement by experts and managers of the company. This provides a simple, understandable description of the interactive process in which individual knowledge is assimilated by organizational knowledge and outstanding individual knowledge rises to organizational knowledge through codification.

\subsection{Organizational Practices. Organizational knowledge} underlies organizational practices, which reflect the organizational strategy and goals [29]. At the same time, organizational actions and their combinations also have a significant impact on the knowledge acquisition of the organization. Managers take different measures to increase the level of organizational knowledge, enhance environmental adaptability, and shape competitive advantages according to the organizational contexts and tasks [30]. In March's model, two typical kinds of organizational practices, socialization and codification, have been examined from the view of organizational learning. Our focus in this study is on the influences of the two on organizational knowledge in different situations.

March models the role of socialization as a process in which individuals modify their beliefs to continue to be consistent with organizational beliefs as a consequence of adaptation to the organization. The organizational knowledge represents the best practice of the organization in the past period. The organization condenses its lessons learned in history into organizational routines and disseminates them in a top-down manner to its members, especially new employees.

In the process of socialization, individuals partially imitate code knowledge, intentionally or unintentionally. Such imperfect imitations can generate an unprecedented combination of knowledge, which may be more consistent with reality than the current knowledge available in the organization. The knowledge of elites, superior individuals, will be reflected in the whole organization through the process of organizational codification and becomes new best practices, which will be learned by other members in the subsequent periods. By coding excellent knowledge of members, the organization improves its current level of knowledge and also the adaptability to the environment.

2.3. Organizational Contexts. In the external settings of the model, March constructs two kinds of organizational contexts, whether the organization exists in an open or closed system and whether the organizational environment is stable or turbulent. The former refers to organizational openness, and the latter refers to environmental dynamism. Considering that the organization is an adaptive system, the former is also defined as system openness. System openness refers to the exchange of energy and information between the system and the outside world. From the perspective of organizational learning, if an organization is open, it means that it can acquire knowledge from the external system. An important but seldom emphasized insight of March's paper [2] is that individuals can learn when participating in an appropriately organized system when they could not do so in isolation. In a closed system, if there are differences in knowledge between individuals, then intraorganizational learning will occur under the guidance of system goals and operating rules. However, intraorganizational learning will accelerate the process of homogenization of individual members' knowledge. When individual members' knowledge tends to be consistent, learning will slow down or even stop. As an adaptive system, learning is a key approach to ensure an organization's adaptability to the environment, especially when the environment is volatile [31]. Once learning stops, the organization may tend to be destroyed.

We cite an organization as an open system if it allows ingress of heterogeneous knowledge from outside the organization [7]. When there is employee turnover in an organization, the replacement individuals bring in heterogeneous knowledge from outside the organization. March [2] implemented an open system characterization by the mechanism of employee turnover, by focusing on the effect of inflow of heterogeneous knowledge via the replacement individuals. There are various kinds of channels for organizations to obtain innovative knowledge, such as engaging with research labs in the universities, attending conferences and trade shows, meeting with industry standards bodies, and engaging external consultants. These approaches change organizational knowledge in two modes. One is a top-down manner, through which the organizational knowledge is changed directly and then spreads in members, such as reformulating the management system of the firm by hiring external experts. The other functions in a down-top manner. In this way, individual members' knowledge is changed first and then the organizational knowledge is influenced indirectly. The typical case is selecting employees to go out to participate in industry training.

In the elaboration above, we use "change" rather than words like "improve" and "refine" because influences from the outside world are not always good. The correctness of organizational knowledge depends on its matching degree with the environment [32]. When the external reality is in turmoil, it is harder to judge whether the replacement of old knowledge is useful for the organization or not. In a turbulent surrounding, the previous best practice of the organization may not work. On the contrary, the knowledge which was unsuitable in the past could turn out to be an effective selection now.

According to the mix of system and environment, the organizational contexts studied in this paper are divided into four categories-stable environments and closed system, 
stable environment and open system, turbulent environment and closed system, and turbulent environment and open system, as shown in Figure 1.

\section{Simulation Model}

In this paper, we recreate March's iconic model with MATLAB R2019a. Four key features of the original model are not modified. All the reported simulation runs are conducted with an organization having fifty members $(n=50)$ where each member has thirty belief dimensions $(m=30)$. A single simulation run is 250 periods $(T=250)$ in length. The only difference is that each of our models is run 10000 times $(I=10000)$, instead of 80 times, in order to avoid errors caused by randomness and enhance the robustness of the results.

\subsection{Model Description}

3.1.1. Subjects in the Model. In the model, there are three subjects, external reality, organizational code, and individual members. The external reality is set as an $m$-dimensional vector, $\mathbf{R}$, representing the environment in which the organization exists. At the beginning of the simulation, each dimension in $\mathbf{R}$ is populated with random values from the set $[-1,+1]$ with equal probability. The organizational code, $\mathbf{O C}$, is also in the form of an $m$-dimensional vector, and each dimension is initialized with the value of "0", signifying "no opinion" on the environment. Individual members, IM, are a matrix with $n$ rows and $m$ columns. Each row in IM represents an organizational member. Each dimension in the row takes a value from the set $[-1,0,+1]$ with the independent probability of $1 / 3$ initially.

3.1.2. Knowledge. Each value of dimension in $\mathbf{R}$ represents the independent belief of a certain aspect of reality. Similarly, each value of dimension in OC and IM reflects the organizational and individual-specific belief about the corresponding dimension of $\mathbf{R}$. If $\mathbf{O C}$ and/or IM have the same value in the corresponding dimension with $\mathbf{R}$, we can say they own correct knowledge in this dimension, representing that they reflect the reality correctly. The level of organizational code knowledge is calculated by dividing the number of dimensions of $\mathbf{O C}$ that match the reality by the total amount of dimensions, $m$. The level of individual knowledge is computed in a similar manner.

3.1.3. Learning Process. The organizational code, $\mathbf{O C}$, learns from superior individual members. In each learning period, OC first identifies the previous-period set of elites, individuals who have more knowledge than the organization. Then, OC determines the majority belief in accordance with the number of different values for each dimension of an elite group. If the value of majority belief is nonzero and $\mathbf{O C}$ has a different belief in the corresponding dimension, OC will update its original belief to the majority one in superior members, with the probability of $p_{2}$, where $p_{2}$ is higher, higher than the strength of the majority. In particular, the probability that a value in a particular dimension in OC will be unchanged at the end of a period is $\left(1-p_{2}\right)^{k}$, where $k$ $(k>0)$ is the number of individuals (within the superior group) who differ from the code on this dimension minus the number who do not. The parameter $p_{2}$ reflects the rate of the learning by $\mathbf{O C}$ from IM. The value of $p_{2}$ is from " 0 " to "1." The larger the value is, the faster the OC learns.

All individual members, IM, learn from the organizational code. In each time step, every individual in IM compares his or her value of each dimension with that of previous-period OC. If their values in the same dimension are not consistent and the value of $\mathbf{O C}$ does not equal " 0 ," then the individual will change its belief to $\mathbf{O C}$, with the probability of $p_{1}$. The parameter $p_{1}$ reflects the rate of learning by the individual in IM from OC. The value of $p_{1}$ is from "0" to "1." The larger the value is, the faster the individual in IM learns.

Changes in organizational contexts: in each run of the model, after the learning process, the set of beliefs of each individual in IM is replaced by a new vector with the probability $p_{3}$, representing the inflow of new knowledge from the outside world. Each value in the new vector is chosen randomly from the set $[-1,0,+1]$ with the independent probability of $1 / 3$. At the same time, each value in $\mathbf{R}$ is changed to its opposite number (from 1 to -1 or from -1 to 1 ), with the probability $p_{4}$.

Equilibrium occurs when all individual members have equivalent knowledge levels, and they share the same belief with the organizational code in each dimension. At this time, the level of organizational code knowledge keeps steady.

3.2. Model Recreation and Verification. According to the subject setting and interaction rules introduced above, we recreate March's model. Figure 2 shows the results of our replication. For each model variant, Figures 2(a) to 2(e) correspond, respectively, to Figures 1 to 5 in March's original study (pp. 76-80). Consistent with March's precedents, the time horizon is through period 250 in Figures 2(a) and 2(b), period 20 in Figures 2(c) and 2(d), and period 100 in Figure 2(e).

Comparing the corresponding curve, including trend and numerical value with March's original results, Figure 1 shows qualitative similarities, which enable us to draw the main conclusions of his paper, but always with quantitative differences, especially in Figure 2(e). Chanda and Miller [33] find the same phenomenon in their paper. They identify, compare, and evaluate March's descriptions of the model and his original program code systematically. As a result, they find that there are three major disparities between the text and the program code. All three features reflected in the program code are in conflict with the logic in March's descriptions. In addition, if these features are dropped, all key conclusions from the original article remain valid. Most relevant existing literature does not involve quantitative comparison with March's original model, just qualitative comparison, even no. According to Chanda and Miller [33], this operation does not guarantee the reconstructed model complete consistency with the original model, which will 


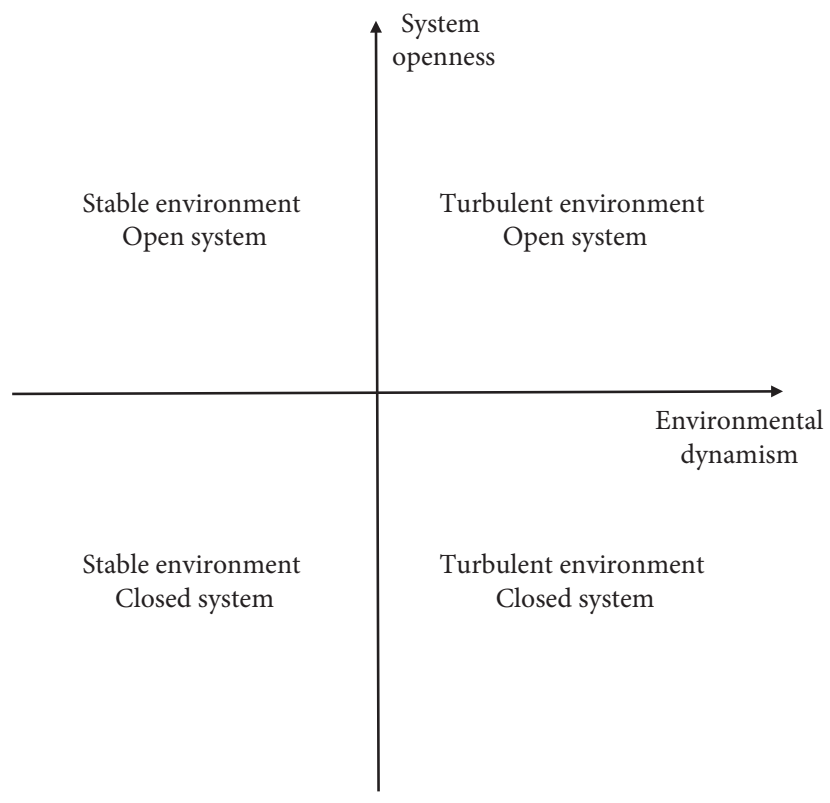

Figure 1: Organizational contexts.

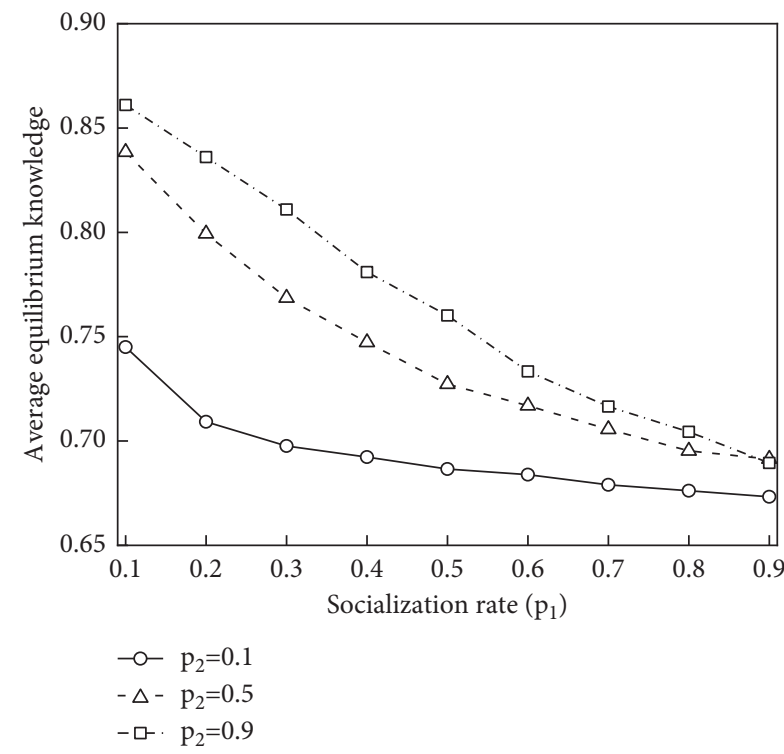

(a)

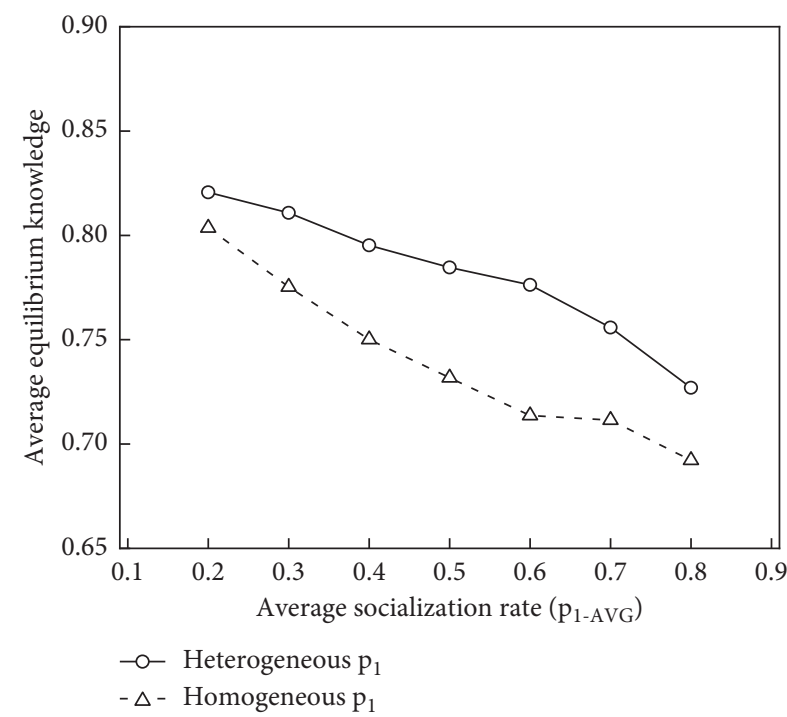

(b)

Figure 2: Continued. 


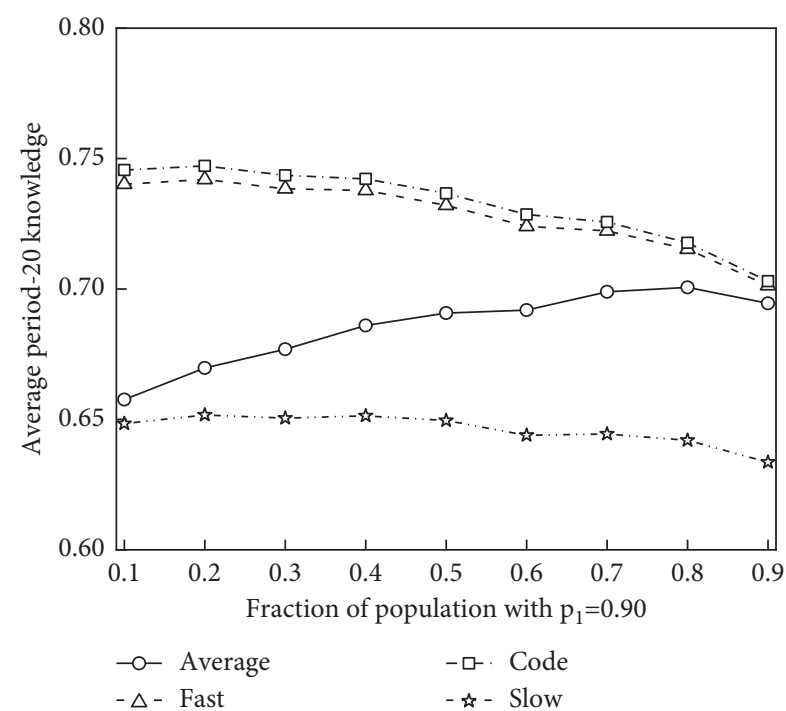

(c)

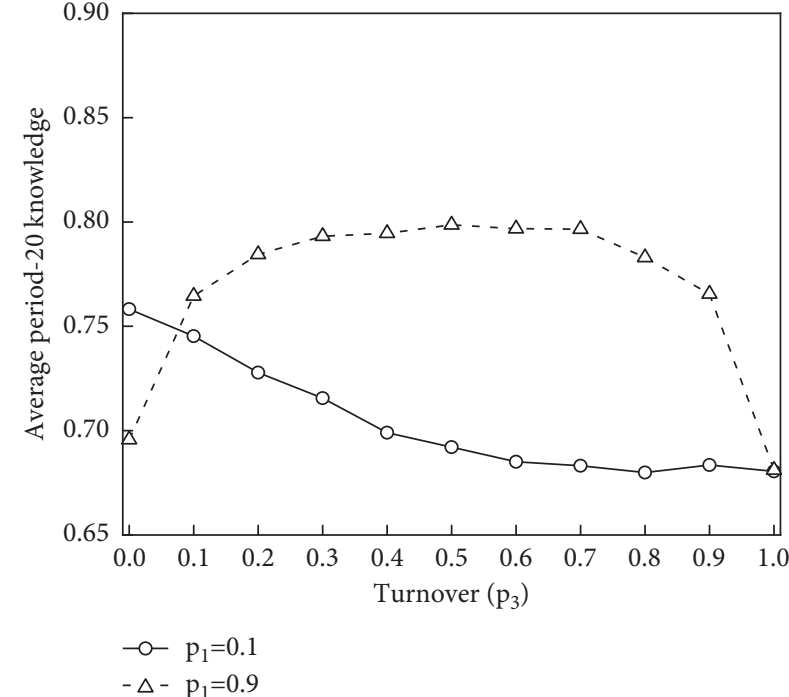

(d)

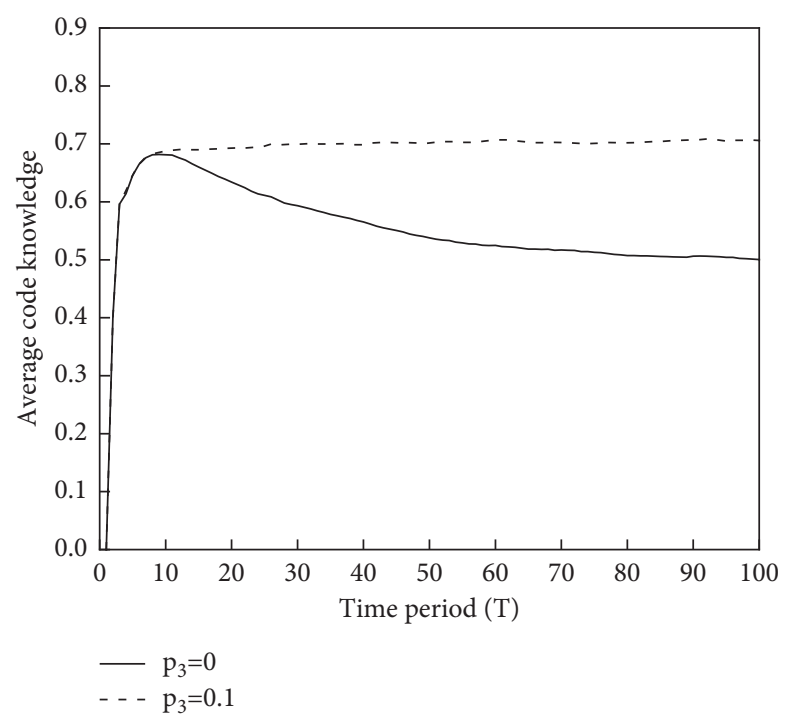

(e)

FIGURE 2: Results of replication. (a) Effect of learning rates $\left(p_{1}, p_{2}\right)$ on equilibrium knowledge. (b) Effect of heterogeneous socialization rates $\left(p_{1}=0.1,0.9\right)$ on equilibrium knowledge. (c) Effect of heterogeneous socialization rates $\left(p_{1}=0.1,0.9\right)$ on period-20 knowledge. (d) Effect of turnover $\left(p_{3}\right)$ and socialization rate $\left(p_{1}\right)$ on period-20 code knowledge. (e) Effect of turbulence $\left(p_{4}\right)$ on code knowledge over time, with and without turnover $\left(p_{3}\right)$.

further influence the robustness of the research conclusion. In conclusion, they propose that the results presented in Figure 1 (pp. 10) should be considered as the baseline for future replication and extension work based on March's model.

Comparing results in Figure 2 with those in Chanda and Miller's [33] Figure 1, all thirteen curves generate consistent, quantitative results, when allowing for small variation produced by random processes across runs of the model. The result of the comparison directly proves the correctness of our model. This is the key guarantee for the reliability of our research conclusions because all the data are generated by the model. The following research is carried out based on the validated model.

\section{Results}

4.1. Stable Environment and Closed System. Figure 3 shows the effects of the socialization rate $\left(p_{1}\right)$ on the level of organizational code knowledge at different codification rates $\left(p_{2}\right)$ over time in the stable environment and closed system. While keeping $p_{2}$ unchanged, the smaller the value of $p_{1}$ is, the more the time it takes for mutual learning between the organizational code and individual members to achieve equilibrium. For example, in Figure 3(a), when $p_{1}$ changes from 0.9 to 0.1 , the corresponding equilibrium time is $15 T, 20 T, 26 T, 31 T$, and more than $50 T$, respectively. In return for a longer learning time to reach equilibrium, a smaller rate of socialization leads to a higher level of equilibrium knowledge. In other words, more 


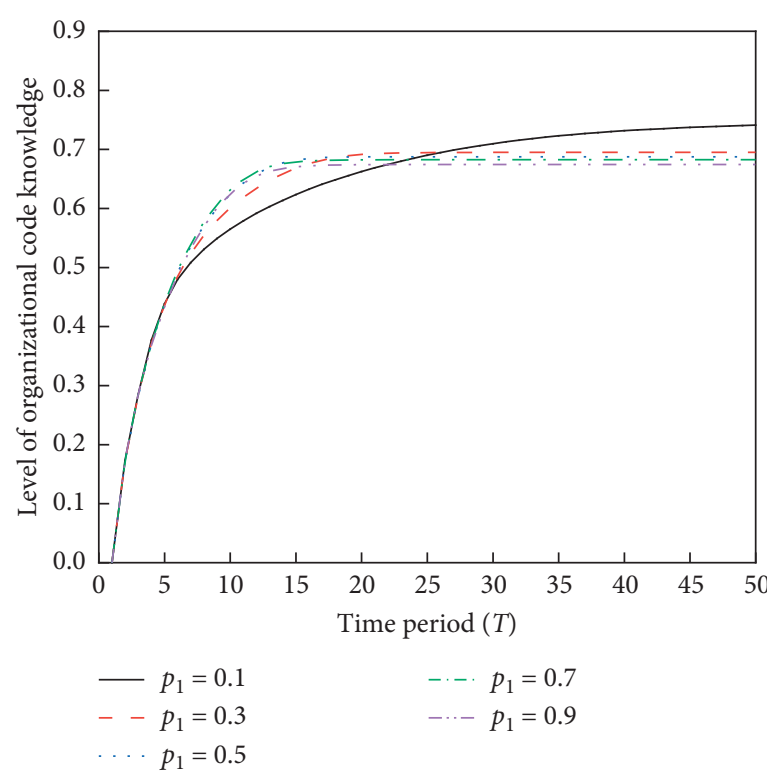

(a)

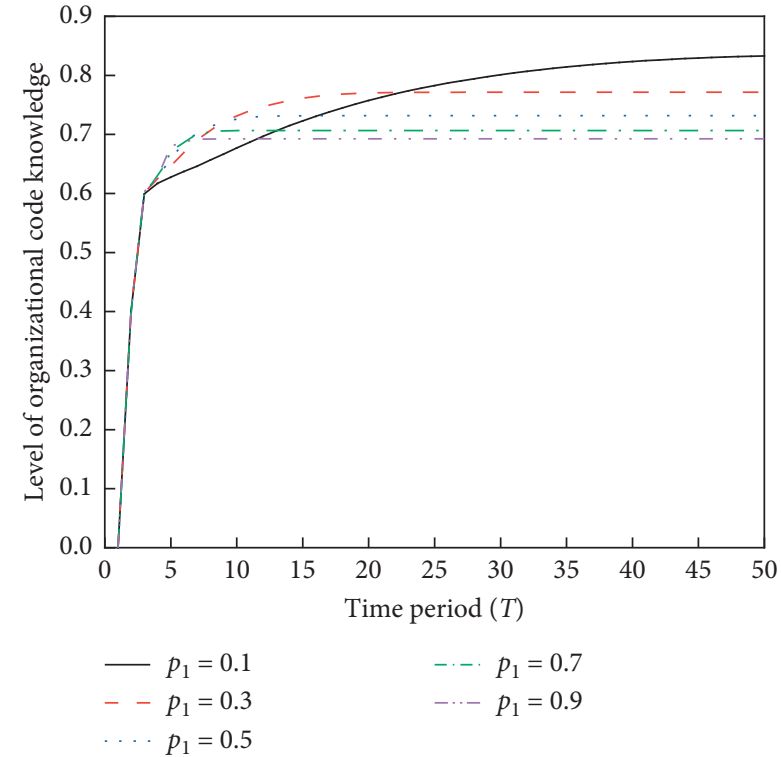

(b)

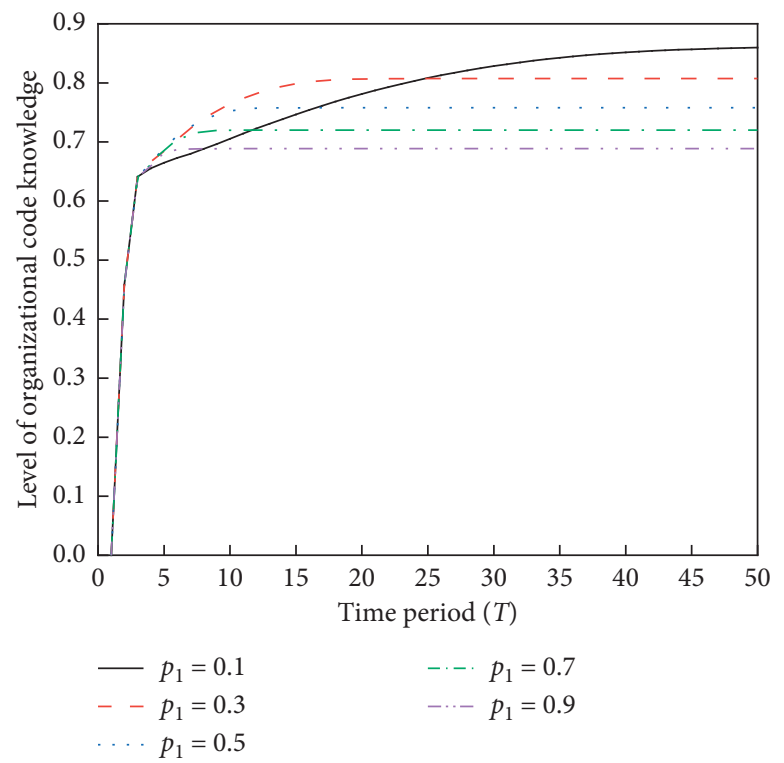

(c)

FIGURE 3: Effects of the socialization rate $\left(p_{1}\right)$ on the level of organizational code knowledge at different codification rates $\left(p_{2}\right)$ over time in the stable environment $\left(p_{4}=0\right)$ and closed system $\left(p_{3}=0\right)$. (a) $p_{2}=0.1$. (b) $p_{2}=0.5$. (c) $p_{2}=0.9$.

rapid socialization brings earlier equilibrium but lower organizational knowledge. This proposition all holds at low (0.1), moderate (0.5), and high (0.9) levels of $p_{2}$.

The essence of socialization is convergence, eliminating knowledge differences between individuals and the organization by adjusting individual beliefs to organizational beliefs. Too rapid socialization makes individuals adopt organizational best practices quickly, which will further make individual beliefs consistent with organizational beliefs in a short time. This finally leads to the result that the organization has no chance to learn because the code can learn only from individuals whose knowledge deviates from it. On the contrary, slower learning maintains the knowledge heterogeneity of members for a longer time, allowing for greater exploration of possible alternatives to improve.

Comparing levels of the organizational code knowledge of the same $p_{1}$ but different $p_{2}$, we can find that larger $p_{2}$ leads to higher knowledge level during the same learning time. In addition, not only the absolute level but also the relative level of the organizational knowledge increases, with the value of $p_{2}$ getting larger. The value of $p_{2}$ reflects the rate of organizational codification. The process of codification is aimed at absorbing mainstream opinions from outstanding groups to improve the knowledge level of the organization. Therefore, the rate of codification directly determines the 
speed of improvement of organizational knowledge. There is a significant positive correlation between the two.

Combining the analysis above, we can make a proposition that when the organization exists in a static environment and there is no inflow of new knowledge from outside, the high level of organizational code knowledge occurs when the organizational code learns rapidly from individual members, and the latter learns slowly from the former. In other words, the combination of the largest codification rate and smallest socialization rate constitutes the best practice of an organization in a stable environment and closed system. Specific to the model, the highest level of organizational code knowledge appears at the position where $p_{1}$ equals 0.1 and $p_{2}$ equals 0.9 .

4.2. Stable Environment and Open System. Figure 4 shows the level of organizational code knowledge at $T=20$ under the abundant combinations of socialization and codification rate, with different degrees of system openness, when organizational learning takes place in an environment without turbulence. Many interesting phenomena could be found by comparing the curves below from different perspectives.

First, when we compare curves under a certain one value of $p_{3}$, we can see that the level of organizational code knowledge increases as the value of $p_{2}$ becomes larger, under the same value of $p_{1}$, which is similar to that in the closed system, as shown in Figure 3. This indicates that speeding up codification is still an effective practice for improving organizational knowledge in an open system.

Second, when the value of $p_{3}$ is not very large, to be precise, smaller than 0.9 , the corresponding curve grows initially and then plateaus. When the value of $p_{3}$ is large enough, greater, or equal to 0.9 , the level of organizational code knowledge shows a monotonically increasing relationship with the socialization rate. This makes clear that the extent of system openness plays a moderating role in the relationship between organizational knowledge and socialization rate, but overall organizational code knowledge levels attained are lower.

Third, the vertex of curves, which represents the highest level of organizational code knowledge, gradually moves to the right as the value of $p_{3}$ increases. When the value of $p_{3}$ equals $0.1,0.3,0.5,0.7$, and 0.9 , the abscissas of the corresponding vertices are $0.4,0.6,0.7,0.8$, and 0.9 , respectively. That is to say, the organization needs more rapid socialization, the rate of which is generally larger than the degree of system openness, to achieve the highest level of code knowledge, when the system is more open. It is easy to understand. System openness refers to the inflow of new knowledge outside, while the socialization rate reflects the learning abilities of individuals. If the speed of influx of new knowledge is greater than that of individual absorption, individuals will not be able to digest this knowledge in time, which will cause waste.

Fourth, with the gradual increase of $p_{3}$, the maximum value of the level of organizational code knowledge increases first and decreases later. When the value of $p_{3}$ changes from 0.1 to 0.9 as shown in the following, the highest knowledge level is $0.846,0.862,0.858,0.845$, and 0.800 , successively. According to this result, we think that the lower or moderate degree of system openness is beneficial for the improvement of organizational knowledge in a stable environment.

Fifth, on the condition that $p_{3}$ is the same, different values of $p_{2}$ correspond to different values of $p_{1}$ to achieve the highest code knowledge. For example, in Figure 4(a), the highest knowledge of each curve reaches, respectively, at the position $p_{1}=0.7$ when $p_{2}=0.1 ; p_{1}=0.2$ when $p_{2}=0.3$ and 0.5 ; $p_{1}=0.4$ when $p_{2}=0.7$ and 0.9. While it comes to Figures $4(\mathrm{a})$, 4(b), and 4(c), combinations of socialization and codification rate of the highest organizational knowledge change. This shows the complex mechanism of the organizational practices and their combinations of organizational knowledge under different constraints.

In order to ensure the reliability of the conclusion, levels of organizational code knowledge at $T=40,60,80$, and 100 are achieved. The results of multidimensional comparisons also support the proposition above.

4.3. Turbulent Environment and Closed System. Figure 5 shows the effects of codification rate $\left(p_{2}\right)$ on the level of organizational code knowledge at different socialization rates $\left(p_{1}\right)$ over time in the context of a turbulent environment and closed system. The values of $p_{4}$ are set to 0.01 , 0.015 , and 0.02 , respectively, representing slight, moderate, and extreme turbulence of the environment. A higher value is not adopted, since a 0.02 value for $p_{4}$ means that the entire reality changes $100 \%$ twice in 100 -time steps, which could be 100 weeks, months, or quarters in the real world. If the reality changes any faster, there is an NIL difference when an organization takes actions based on learning (there is nothing to learn; most knowledge becomes obsolete very quickly) vis-a-vis when the organization takes random actions.

Overall, if the organization is in a closed atmosphere, then the turbulence outside will have a significant, negative impact on its knowledge level. The more turbulent the environment is, the lower the level of code knowledge is when other conditions keep consistent. The strength of this negative influence is related to the socialization rate. When the socialization rate is small, differences in knowledge caused by various levels of environmental turbulence (curves with a different color) are very apparent. With an increase in the socialization rate, differences in knowledge become smaller and smaller. This phenomenon is more pronounced in the environment with moderate-level and high-level turbulence than low-level turbulence. In addition, the number of learning periods is also a key factor influencing knowledge differences in this circumstance.

Increasing environmental turbulence makes the organization's original best practices no longer applicable. In this case, the organization must strengthen learning to be able to improve adaptability. Too rapid socialization drives out the heterogeneity of members' knowledge quickly. As a result, the organization loses the chance to learn in a short time. With the increase of learning time, the mutual learning between the organizational code and individual members 

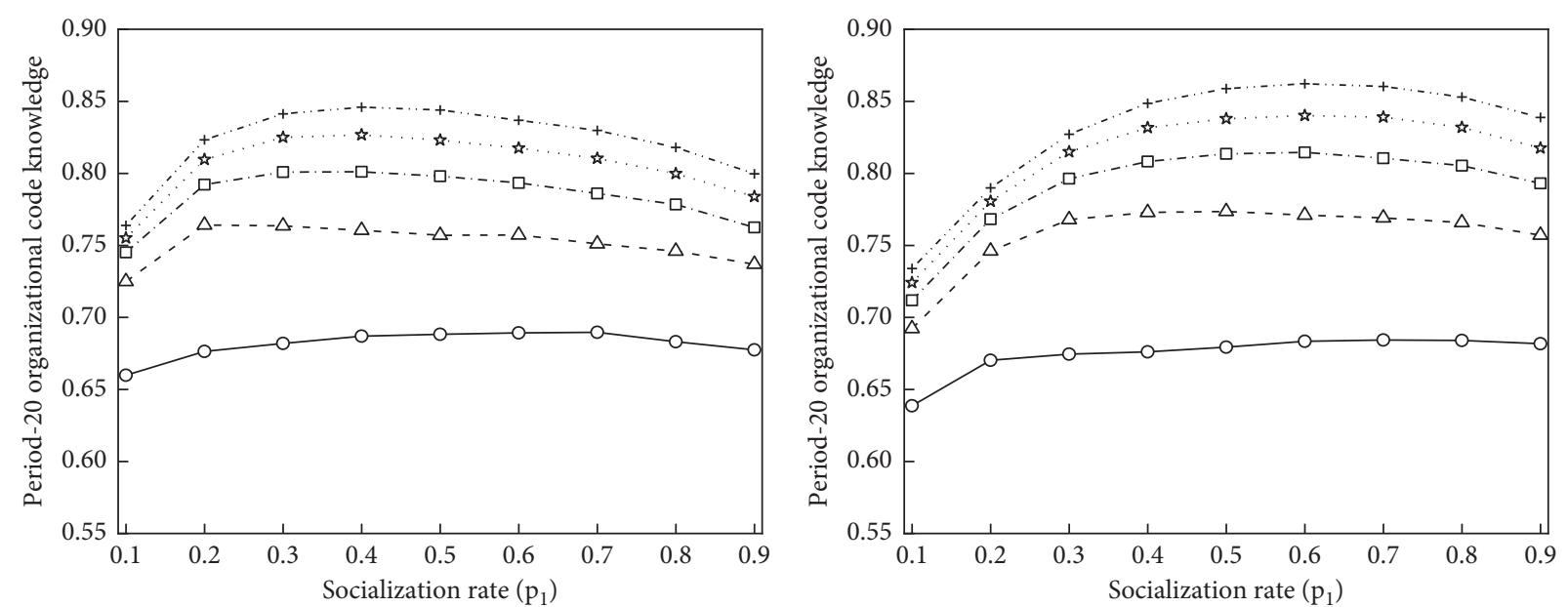

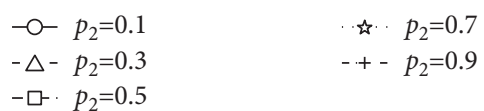

(a)

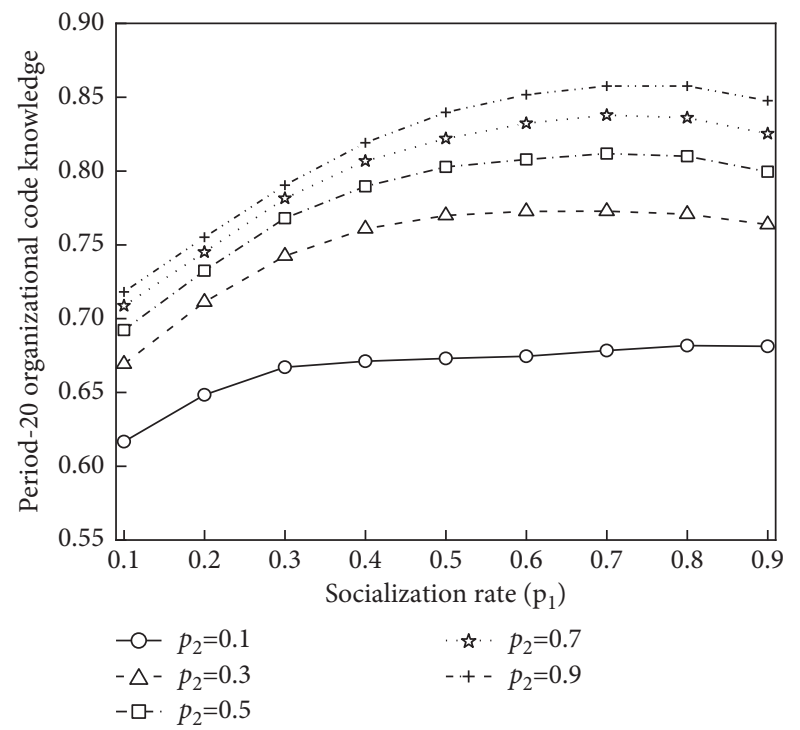

- $-p_{2}=0.1$

$-\triangle-p_{2}=0.3$

-ㅁ. $p_{2}=0.5$

ㄴ. $p_{2}=0.7$

- + - $p_{2}=0.9$

(b)

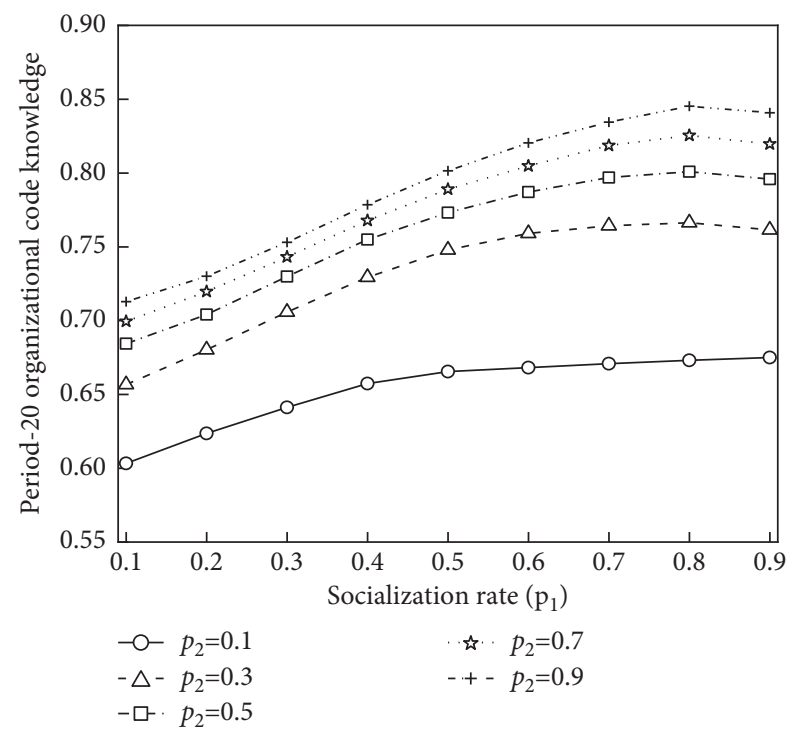

(c)

(d)

Figure 4: Continued. 


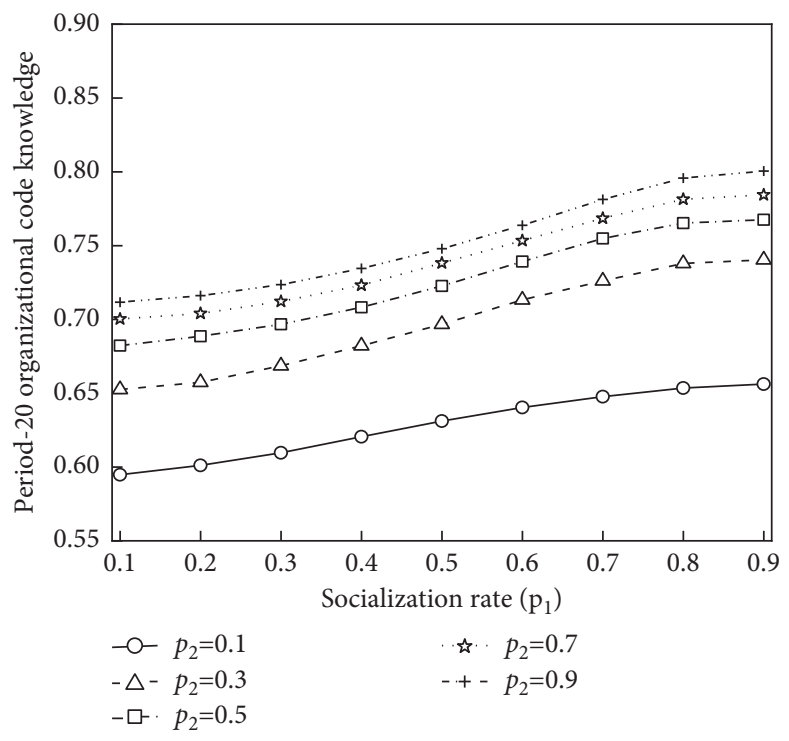

(e)

Figure 4: Effects of combinations of the socialization rate $\left(p_{1}\right)$ and codification rate $\left(p_{2}\right)$ on the organizational knowledge with different degrees of system openness in the stable environment $\left(p_{4}=0\right)$. (a) $p_{3}=0.1$. (b) $p_{3}=0.3$. (c) $p_{3}=0.5$. (d) $p_{3}=0.7$. (e) $p_{3}=0.9$.
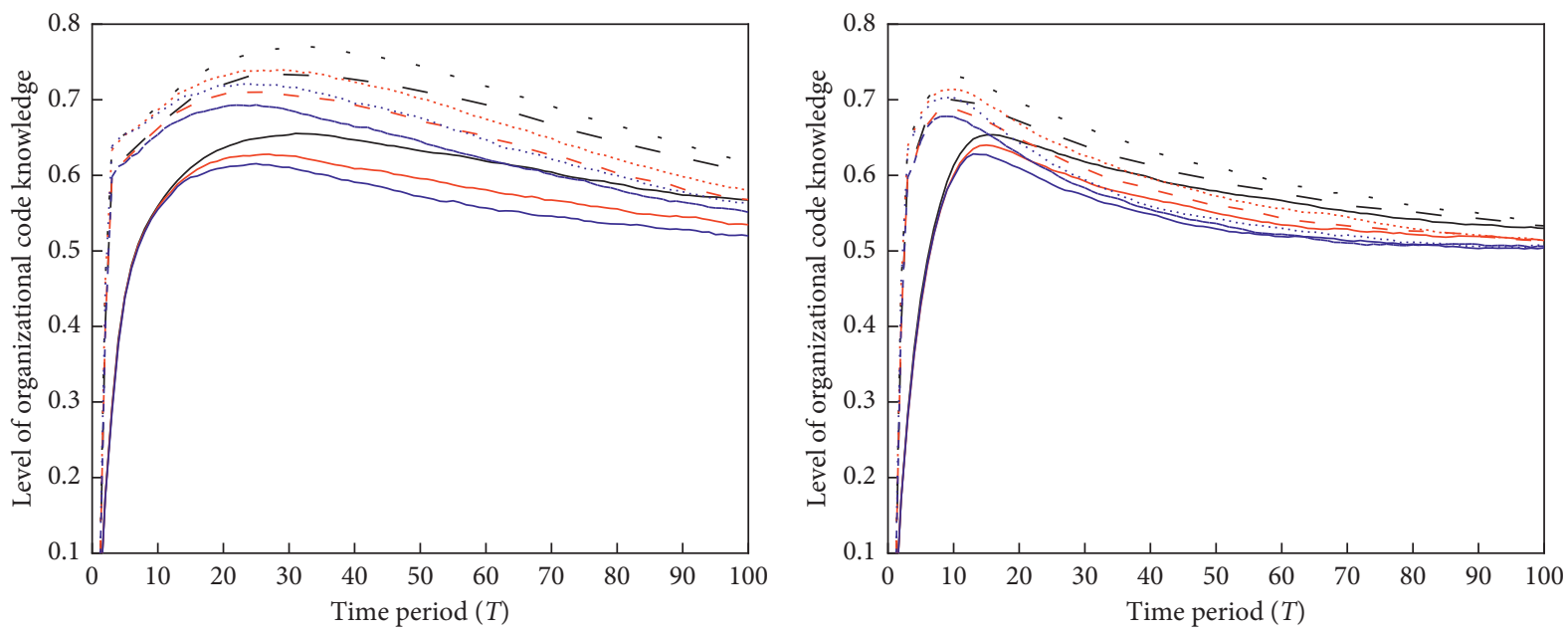

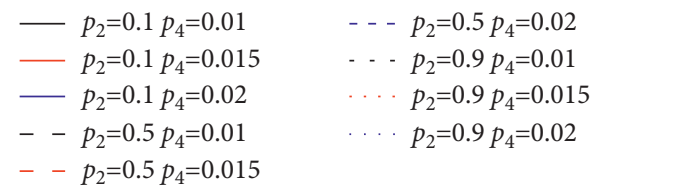

(a)

$$
\begin{aligned}
-p_{2}=0.1 p_{4}=0.01 & \cdots-p_{2}=0.5 p_{4}=0.02 \\
-p_{2}=0.1 p_{4}=0.015 & \cdots-p_{2}=0.9 p_{4}=0.01 \\
-p_{2}=0.1 p_{4}=0.02 & \cdots p_{2}=0.9 p_{4}=0.01 \\
-p_{2}=0.5 p_{4}=0.01 & \cdots p_{2}=0.9 p_{4}=0.02 \\
-p_{2}=0.5 p_{4}=0.015 &
\end{aligned}
$$

(b)

Figure 5: Continued. 


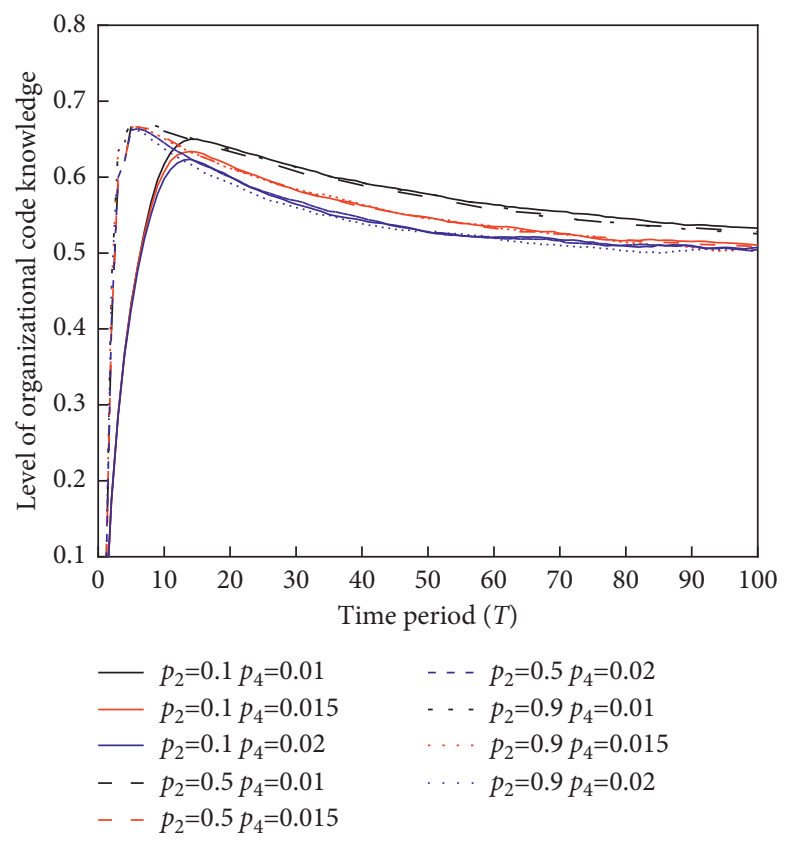

(c)

FigURE 5: Effects of codification rate $\left(p_{2}\right)$ on the level of organizational code knowledge at different socialization rates $\left(p_{1}\right)$ over time in the turbulent environment and closed system $\left(p_{3}=0\right)$. (a) $p_{1}=0.1$. (b) $p_{1}=0.5$. (c) $p_{1}=0.9$.

gradually approaches the equilibrium state, making knowledge differences disappear piece by piece.

When the extent of environmental turbulence is low and moderate, the level of organizational code knowledge improves with the increase of the codification rate. While the environment is highly turbulent, the former decreases with the increase of the latter. As the socialization rate gets large, not only does the maximum value of code knowledge become small, but also knowledge differences caused by various codification rates (curves with the same color) disappear gradually. In addition, from a fine-grained view, when the environmental turbulence is at a low and moderate level, the level of code knowledge first increases, then decreases, and finally stabilizes as the learning cycle increases.

When the organization is in a turbulent environment and closed system, the larger codification rate could bring the improvement to the level of organizational code knowledge, but this is temporary. As mutual learning continues, the effect brought by efforts of increasing the codification rate will gradually weaken. Under the circumstance that the socialization rate is high, accelerating codification will lose its effect after a short period of time, even in the slightly turbulent environment, let alone a more dynamic one.

4.4. Turbulent Environment and Open System. Figure 6 shows the effects of different combinations of the extent of environmental dynamism and system openness on the level of organizational code knowledge over time when rates of socialization and codification are both moderate $\left(p_{1}=0.5 ; p_{2}=0.5\right)$. The negative effects of environmental turbulence on the level of organizational code knowledge still exist. However, when the system openness is added, the situation becomes a little complex. In the case of moderatelevel $\left(p_{4}=0.015\right)$ and high-level $\left(p_{4}=0.02\right)$ environmental turbulence, the level of organizational code knowledge first increases and then decreases as the system becomes more open. On the contrary, if the environment is turbulent sightly $\left(p_{4}=0.01\right)$, the level of organizational code knowledge will decrease with the system openness getting large.

Considering that the organizational environment is objective, but the openness of the organization can be subjectively adjusted, which depends on the organization's strategic decision-making level, top managers of the organization need to adopt different organizational external strategies to deal with different levels of chaos in the environment. From the results above, it appears that it is a favorable alternative for organizations to remain moderately open, regardless of the level of turbulence in the environment.

In addition, when comparing the degree of proximity of the organizational code knowledge under different openness levels but the same dynamism level, we can further provide some practical suggestions for the organization. When the environment is moderately turbulent, the senior managers may choose practices to facilitate moderate-level, rather than high-level openness of the organization, because the two have a similar outcome, but the latter will make the organization put in more effort. Any organizational practice requires a certain cost. Generally speaking, increasing the openness of the organization has more difficult operations and higher costs than reducing it in practice. If the organization allocates $50 \%$ of the resources to achieve $90 \%$ of the final effect, then this will be a major benefit to the sustainable 


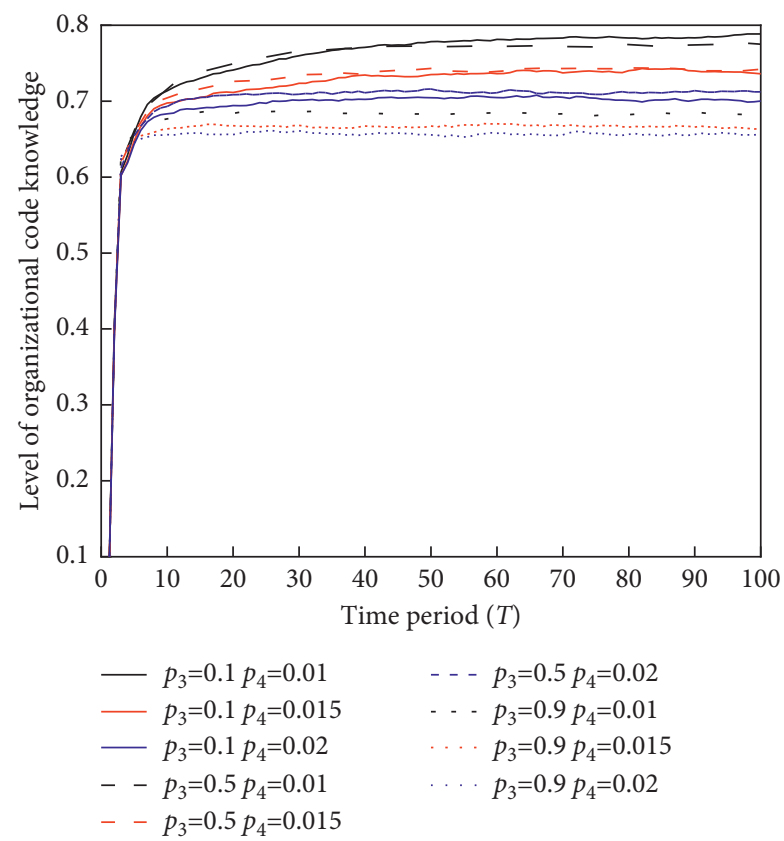

Figure 6: Effects of different combinations of the extent of environmental dynamism and system openness on organizational code knowledge over time.

development of the organization, although it does not achieve the ideal best practice.

In order to ensure the reliability of the conclusion, various combinations of socialization and codification rates are experimented with to avoid the fortuity of the qualitative relationship. Results of experimentations show that different rates of socialization and codification do not affect the establishment of conclusions above. Therefore, similar figures do not have to have an additional display here.

\section{Discussion}

This paper revisits March's original model and pays attention to the effects of practice mix on organizational knowledge. The obtained conclusions can provide helpful references for follow-up researches in this domain. Despite this, considering that the four main conclusions gained in the paper are all based on the calculation results of the model, there are some aspects related to them worth discussing, especially challenges in how to apply these theoretical deductions to organizational operations.

In different organizational contexts, whether the environment is turbulent or not and whether the organization is in an open system or not, speeding up organizational codification $\left(p_{2}\right)$ is an effective option for improving the level of organizational knowledge. Then, how to facilitate members to enrich organizational codes, which is essentially creating new knowledge, becomes an important issue, especially for organizational managers. In this regard, McKinsey \& Company's evolving knowledge management processes [34] may provide some insight. First, daily documentation of concepts, rather than just verbal communication, facilitates the internal transfer of ideas and the capture of internally developed knowledge. This is an effective tool for generating fresh knowledge since the really big ideas are usually sorted out by the internal market. Second, building a common database of knowledge is a recommended practice. With that, a full-time database maintenance staff is essential, not only to monitor the quality of the data but also to help members access relevant information more easily. Third, the training of organizational members should be reemphasized. The influx of external knowledge not only contributes to the development of members' technical and professional skills but also can force employees to reexamine what they take for granted. On the other hand, wisdom from the East reminds us that creating new knowledge is not just a matter of processing all kinds of objective information, but a process of transforming tacit knowledge into explicit [35]. One of the important tasks of managers is to tap into the highly subjective intuitions and hunches of individual employees and then make them available for testing and use by the organization. Of course, this presupposes a high level of employee identification with the firm and its mission.

As stated above, the essence of the system openness is explorative learning, which refers to the inflow of new knowledge outside. The system openness can be controlled by organizational decision-makers and managers. They can allocate organizational resources in engaging with research labs in the university, attending conferences and trade shows, meeting with industry, hiring external consultants, and so forth, to access new knowledge. In addition, personnel turnover can also bring new knowledge by means of replacement individuals who have acquired knowledge from sources outside the organization. Explorative learning is very necessary for the long-term development of the organization, especially in a turbulent environment [36]. However, compared with exploitative learning, whose focus is on the 
refinement of old knowledge, explorative learning has characteristics of high cost, high risk, and high uncertainty, like disruptive innovation in the field of innovative research. Also, it takes more time than exploitative learning for managers to observe its impacts on the organizational performance, though it usually improves the organizational performance greatly, if successful. This comes back to March's central thesis through his classic model, which is that "maintaining an appropriate balance between exploration and exploitation is a primary factor in organizational survival and prosperity." Exploitative learning guarantees organizational existence, and explorative learning facilitates organizational development.

This paper focuses on March's original model itself and obtains some useful conclusions, which can be regarded as the foundation for relative researches in the future. There are some interesting aspects relative to this paper worth further studying. Firstly, this paper shows that how organizational practices affect organizational knowledge in different contexts. This belongs to the macrolevel research category. In order to have a deeper understanding of the model, the complex influence mechanism and process of organizational practices and contexts on organizational knowledge remain to be studied from the microlevel. Secondly, we mention in the paper that various organizational practices require different resource consumption. Considering the marginal effect of organizational costs, the best performance may not respond to the best cost performance. What is discussed in the paper is the best performance (highest knowledge level); when it comes to the best cost performance, the present conclusions may be different. Thirdly, existing studies extend March's model from various aspects, like learning process, knowledge feature, environmental characteristic, organizational structure, and so forth. Checking whether the conclusions in the paper are valid under the new research design and context may be regarded as a prospect of future researches.

\section{Conclusions}

In summary, we recreate March's classic agent-based model perfectly in this research. On the basis of the reconstructed model, impacts of practice combinations on the level of organizational knowledge in different contexts are studied systematically. The conclusions of the research are shown as follows:

(1) In a stable environment and closed system, the highest level of organizational equilibrium knowledge can be achieved when the organization learns from individuals fastest and individuals learn from the organization with their smallest rates.

(2) To acquire the highest level of organizational knowledge in a stable environment and open system, the largest codification rate is necessary, and the socialization rate should be larger than the degree of the system openness.

(3)When organizational learning occurs in a turbulent environment and closed system, accelerating socialization and codification can both improve the level of organizational knowledge in a short time but will lose influence with the increase of learning time.

(4) The increase of the extent of system openness can relieve, to a certain degree, the negative correlation between the environmental turbulence and the level of organizational knowledge.

\section{Data Availability}

The program details and data used to support the findings of this study are available from the corresponding author upon request.

\section{Conflicts of Interest}

The authors declare that there are no conflicts of interest regarding the publication of this paper.

\section{Acknowledgments}

This research was funded by the National Natural Science Foundation of China (71871164).

\section{References}

[1] T. Kim and M. Rhee, "Exploration and exploitation: internal variety and environmental dynamism," Strategic Organization, vol. 7, no. 1, pp. 11-41, 2009.

[2] J. G. March, "Exploration and exploitation in organizational learning," Organization Science, vol. 2, no. 1, pp. 71-87, 1991.

[3] S. S. Chanda, "Inferring final organizational outcomes from intermediate outcomes of exploration and exploitation: the complexity link," Computational and Mathematical Organization Theory, vol. 23, no. 1, pp. 61-93, 2017.

[4] R. Wilden, J. Hohberger, T. M. Devinney, and D. Lavie, "Revisiting James march (1991): whither exploration and exploitation?" Strategic Organization, vol. 16, no. 3, pp. 352-369, 2018.

[5] S. S. Chanda and S. Ray, "Optimal exploration and exploitation: the managerial intentionality perspective," Computational and Mathematical Organization Theory, vol. 21, no. 3, pp. 247-273, 2015.

[6] S. S. Chanda, S. Ray, and B. McKelvey, "The continuum conception of exploration and exploitation: an update to march's theory," Management, vol. 21, no. 3, pp. 1032-1079, 2018.

[7] S. S. Chanda and B. McKelvey, "Back to the basics: reconciling the continuum and orthogonal conceptions of exploration and exploitation," Computational and Mathematical Organization Theory, vol. 26, no. 2, pp. 175-206, 2020.

[8] S. Rodan, "Exploration and exploitation revisited: extending march's model of mutual learning," Scandinavian Journal of Management, vol. 21, no. 4, pp. 407-428, 2005.

[9] S. Blaschke and D. Schoeneborn, "The Forgotten function of forgetting: revisiting exploration and exploitation in organizational learning," Soziale Systeme, vol. 12, no. 1, pp. 100-120, 2006.

[10] K. D. Miller, M. Zhao, and R. J. Calantone, “Adding interpersonal learning and tacit knowledge to march's explorationexploitation model," Academy of Management Journal, vol. 49, no. 4, pp. 709-722, 2006. 
[11] K. D. Miller and D. Martignoni, "Organizational learning with forgetting: reconsidering the exploration-exploitation tradeoff," Strategic Organization, vol. 14, no. 1, pp. 53-72, 2016.

[12] C. Fang, J. Lee, and M. A. Schilling, "Balancing exploration and exploitation through structural design: the isolation of subgroups and organizational learning," Organization Science, vol. 21, no. 3, pp. 625-642, 2010.

[13] M. A. Schilling and C. Fang, "When hubs forget, lie, and play favorites: interpersonal network structure, information distortion, and organizational learning," Strategic Management Journal, vol. 35, no. 7, pp. 974-994, 2014.

[14] G. C. Kane and M. Alavi, "Information technology and organizational learning: an investigation of exploration and exploitation processes," Organization Science, vol. 18, no. 5, pp. 796-812, 2007.

[15] S. Park, A. Stylianou, C. Subramaniam, and Y. Niu, "Information technology and interorganizational learning: an investigation of knowledge exploration and exploitation processes," Information \& Management, vol. 52, no. 8, pp. 998-1011, 2015.

[16] H. Zhang, Y. Xi, and W. B. Lee, "Exploration and exploitation in parallel problem solving: effect of imitation strategy and network structure," Systems Approaches to Knowledge Management, Transfer, and Resource Development, pp. 201-215, 2012.

[17] N. Siggelkow and J. W. Rivkin, "When exploration backfires: unintended consequences of multilevel organizational search," Academy of Management Journal, vol. 49, no. 4, pp. 779-795, 2006.

[18] D. Lazer and A. Friedman, "The network structure of exploration and exploitation," Administrative Science Quarterly, vol. 52, no. 4, pp. 667-694, 2007.

[19] H. E. Posen and D. A. Levinthal, "Chasing a moving target: exploitation and exploration in dynamic environments," Management Science, vol. 58, no. 3, pp. 587-601, 2012.

[20] J. Bendor, T. M. Moe, and K. W. Shotts, "Recycling the garbage can: an assessment of the research program," American Political Science Review, vol. 95, no. 1, pp. 169-190, 2001.

[21] R. Adner, L. Pólos, M. Ryall, and O. Sorenson, “The case for formal theory introduction to special topic forum," Academy of Management Review, vol. 34, no. 2, pp. 201-208, 2009.

[22] G. Fioretti, "Agent-based simulation models in organization science," Organizational Research Methods, vol. 16, no. 2, pp. 227-242, 2013.

[23] J. R. Harrison, Z. Lin, G. R. Carroll, and K. M. Carley, "Simulation modeling in organizational and management research," Academy of Management Review, vol. 32, no. 4, pp. 1229-1245, 2007.

[24] R. E. Ployhart and T. P. Moliterno, "Emergence of the human capital resource: a multilevel model," Academy of Management Review, vol. 36, no. 1, pp. 127-150, 2011.

[25] R. G. Rocha and P. G. Pinheiro, "Organizational spirituality and knowledge management supporting organizational practical wisdom," Spirituality Studies, vol. 7, no. 1, pp. 68-83, 2021.

[26] J. Sun, X. Ren, and C. J. Anumba, "Analysis of knowledgetransfer mechanisms in construction project cooperation networks," Journal of Management in Engineering, vol. 35, no. 2, 2019.

[27] C. I. Sepulveda-Rivillas, J. Alegre, and V. Oltra, "Impact of knowledge-based organizational support on organizational performance through project management," Journal of Knowledge Management, 2021.
[28] X. Zhang, Y. Le, Y. Liu, and X. Chen, "Fostering ambidextrous innovation strategies in large infrastructure projects: a team heterogeneity perspective," IEEE Transactions on Engineering Management, 2021.

[29] R. G. C. Dirksen, "Organisationsentwicklung durch Markenstrategie,” Gruppe. Interaktion. Organisation, vol. 52, pp. 525-536, 2021.

[30] R. K. G. Potnuru, C. K. Sahoo, and K. C. Parle, "HRD practices, employee competencies and organizational effectiveness: role of organizational learning culture," Journal of Asia Business Studies, vol. 15, no. 3, pp. 401-419, 2021.

[31] F. R. Augusto, "Food assistance in Portugal: organizational challenges in three different contexts," Journal of Organizational Ethnography, 2021.

[32] C. Froehlich, "Innovation and sustainability in the organizational context," Gestao E Desenvolvimento, vol. 18, no. 1, p. 2, 2021.

[33] S. S. Chanda and K. D. Miller, "Replicating agent-based models: revisiting march's exploration-exploitation study," Strategic Organization, vol. 17, no. 4, pp. 425-449, 2019.

[34] C. A. Bartlett, McKinsey \& Company: Managing Knowledge and Learning, Harvard Business School Press, Cambridge, MA, USA, 1999.

[35] I. Nonaka, "The knowledge-creating company," Harvard Business Review, vol. 85, no. 7-8, p. 162, 2007.

[36] X. Zhang, Y. Le, Y. Liu, and M. Liu, "Fostering ambidextrous innovation in infrastructure projects: differentiation and integration tactics of cross-functional teams," Journal of Construction Engineering and Management, vol. 147, no. 6, Article ID 04021046, 2021. 